$E_{1}-V$ is finite. But $\tau^{\prime}$ is a $T_{1}$-topology so there is a $U^{*} \in \tau^{\prime}$ such that $\varnothing \neq U^{*} \subset U$ and $U^{*} \cap E_{1}=\varnothing$. Thus $U^{*} \cap E_{2}=U^{*}$ and $U^{*} \in \tau \wedge \tau^{\prime}$ but $U^{*} \notin \mathrm{C}$. Thus if $\tau \vee \tau^{\prime}=1$ then $\tau \wedge \tau^{\prime} \neq \mathrm{e}$. Hence it has been verified that

ThEOREM 2. The lattice $\Lambda$ of $T_{1}$-topologies on an infinite set $E$ is not complemented.

\title{
REFERENCES
}

1. R. W. Bagley, On the characterization of the lattice of topologies, J. London Math. Soc. 29-30 (1954-1955), 247-249.

2. O. Fröhlich, Das Halbordnungssystem der topologischen Räume auf einer Menge, Math. Ann. 156 (1964), 79-95.

3. J. Hartmanis, On the lattice of topologies, Canad. J. Math. 10 (1958), 547-553.

4. A. K. Steiner, The lattice of topologies: structure and complementation, Trans. Amer. Math. Soc. (to appear).

University of New Mexico and

Texas Technological College

\section{SPACES WITH ACYCLIC POINT COMPLEMENTS}

\section{MICHAEL C. MCCORD}

1. Introduction. All homology groups will be singular homology with integer coefficients, reduced in dimension zero. If $0 \leqq n \leqq \infty$, a space $X$ is $n$-acyclic if $H_{q}(X)=0$ for all integers $q \leqq n$.

Definition. A Hausdorff space $M$ is an $A^{n}$-space if the complement of each point in $M$ is $n$-acyclic.

The condition on a point $x$ in $M$ that $M-x$ be $n$-acyclic is similar to the notion that $x$ be a non- $r$-cut point $(r \leqq n)$, defined by R. L. Wilder [9, p. 218], using Čech theory.

Clearly spheres are $A^{\infty}$-spaces. The object of this paper is to investigate to what extent $A^{n}$-spaces are like spheres. I wish to thank W. S. Massey for useful suggestions.

2. Statement of results. Examples. Open cells or closed cells of dimension $n+2$ are clearly $A^{n}$-spaces. Hilbert space $l^{2}$ is an $A^{\infty}$-space; in fact by a theorem of Klee [5, p. 22], the complement of every compact subset of $l^{2}$ is homeomorphic to $l^{2}$ itself.

Received by the editors October 26, 1965. 
A polyhedron $M$ is a homology n-manifold if and only if at all points $x$ of $M$ the local homology groups $H_{q}(M, M-x)$ are the same as those of Euclidean $n$-space. Every such space which is also a homology $n$ sphere must be an $A^{\infty}$-space. This may be proved directly by showing that for each $x$ in $M$ the homomorphism $H_{*}(M) \rightarrow H_{*}(M, M-x)$ in the exact sequence of the pair $(M, M-x)$ is an isomorphism. For examples of such spaces for $n \geqq 3$ besides spheres one may take suspensions of Poincaré spaces [7, p. 218].

Let us now give an example, for each nonnegative integer $n$, of a compact $(n+1)$-dimensional polyhedron which is an $A^{n}$-space but which is neither a homology manifold nor a homology sphere. For this we need

TheOREM 1. If $M$ is an $A^{n}$-space containing more than one point, then the suspension $S(M)$ of $M$ is an $A^{n+1}$-space.

Now let $\theta$ be a theta curve (a circle with a diameter). Clearly $\theta$ is an $A^{0}$-space. Thus by Theorem 1 , the $n$-fold suspension $S^{(n)}(\theta)$ of $\theta$ is an $A^{n}$-space. In dimension $n+1$, the homology group of $S^{(n)}(\theta)$ and, at some points, the local homology group of $S^{(n)}(\theta)$, are free abelian of rank 2 .

In contrast to this example, the following theorem shows that things are nicer when the dimension of a polyhedral $A^{n}$-space is equal to $n$.

THEOREM 2. If $M$ is an n-dimensional polyhedron which is an $A^{n_{-}}$ space, then $M$ is a homology manifold and a homology sphere.

For dimensions less than 3 we get the following characterization, which is similar to those in [9, pp. 220-223] using Cech theory.

Theorem 3. If $0 \leqq n \leqq 2$ and $M$ is an $A^{n}$-space having $H_{n}(M) \neq 0$, then $M$ is a topological $n$-sphere.

This follows from Theorem 2 in case $M$ is a polyhedron. However note that the only point-set assumption put on $M$ is that it be a Hausdorff space. This condition is crucial; for it is shown in [6] that for each $n \geqq 0$ there exists a finite $T_{0}$ space (having $2 n+2$ points) which has the same (singular) homology groups and homotopy groups as the $n$-sphere and in which the complement of each point is $\infty$ acyclic.

The sphere-like character of $A^{n}$-spaces is illustrated by the following theorem, which generalizes that part of the Alexander duality theorem dealing with the complement of a cell or of a sphere in a sphere. 
Theorem 4 . Let $0 \leqq k \leqq n \leqq \infty$ and let $M$ be an $A^{n}$-space.

(a) If $D$ is a closed $k$-cell in $M$, then $M-D$ is $(n-k)$-acyclic.

(b) If $S_{0}^{k}$ is a k-sphere in $M$, then $H_{p}\left(M-S_{0}^{k}\right) \approx H_{p+k+1}(M)$ whenever $0 \leqq p<n-k$.

The argument is analogous to the line of reasoning in [1] and also overlaps with the proof in [2] of the corresponding part of the Alexander duality theorem. Examples will be given to show that in part (a) we cannot assert that $M-D$ is $(n-k+1)$-acyclic and that in part (b) we do not obtain the isomorphism for $p=n-k$.

3. Notation for the proofs. For each $n \geqq 0$ let $R^{n}$ be Euclidean $n$-space, consisting of all infinite sequences $\left(x_{1}, x_{2}, \ldots\right)$ of reals such that $x_{i}=0$ for $i>n$. (Thus $R^{n} \subset R^{n+1}$.) In $R^{n}$ let $D^{n}$ be the unit disk $\{x:\|x\| \leqq 1\}$ and let $S^{n-1}$ be its boundary. The standard $n$-cube $I^{n}$ is the $n$-fold product of the unit interval $I=[0,1]$.

4. Proof of Theorem 1. Let $\nu: M \times I \rightarrow S(M)$ be the quotient map, identifying $M \times 0$ and $M \times 1$ to the poles $p_{0}$ and $p_{1}$, respectively. The complement of either $p_{0}$ or $p_{1}$ is contractible. Hence consider the complement of $y=\nu(x, t)$, where $0<t<1$. In the proper triad

$$
(S(M)-y ; \nu(M \times[0, t])-y, \nu(M \times[t, 1])-y),
$$

the second and third members are contractible, their union is the first member, and their intersection is homeomorphic to $M-x$, which is nonempty. Hence the (reduced) Mayer-Vietoris sequence of this triad gives the isomorphism $H_{q}(M-x) \approx H_{q+1}(S(M)-y)$, all $q$.

5. Proof of Theorem 2. For each point $x$ of $M$, the group $H_{q}(M-x)$ is zero for $q \leqq n$ since $M$ is an $A^{n}$-space, and is zero for $q>n$ since $M$ is an $n$-dimensional polyhedron. Hence from the exact sequence of the pair $(M, M-x)$, we see that $H_{*}(M, M-x) \approx H_{*}(M)$. On the other hand, choose some point $x_{0}$ in a principal simplex of $M$, so that $x_{0}$ has a neighborhood $V \equiv R^{n}$. Thus $H_{*}(M) \approx H_{*}\left(M, M-x_{0}\right)$ $\approx H_{*}\left(V, V-x_{0}\right) \approx H_{*}\left(R^{n}, R^{n}-0\right) \approx H_{*}\left(S^{n}\right)$.

6. Proof of Theorem 4. (a) The proof is by induction on the dimension $k$ of $D$, the case $k=0$ holding since $M$ is an $A^{n}$-space. Suppose that $k$ is a positive integer $\leqq n$ and that the result holds for $(k-1)$ cells. Let $D$ be the image of an imbedding $h$ of $I^{k}$ into $M$. Suppose $0 \leqq q \leqq n-k$ and suppose there exists a nonbounding $q$-cycle $z$ in $M-D$. Bisect $D$ as follows. Consider the $k$-cells $D_{1}=h([0,1 / 2]$ $\left.\times I^{k-1}\right), \quad D_{2}=h\left([1 / 2,1] \times I^{k-1}\right)$, and the $(k-1)$-cell $D_{0}=h(\{1 / 2\}$ $\left.\times I^{k-1}\right)$. Since $q+1 \leqq n-(k-1)$, the inductive assumption and the 
Mayer-Vietoris sequence of the proper triad $\left(M-D_{0} ; M-D_{1}, M-D_{2}\right)$ show us that either $z \times 0$ in $M-D_{1}$ or $z \times 0$ in $M-D_{2}$. (This triad is proper because its members are open subsets of $M$; see [3, p. 34, p. 199].) A continuation of the bisection process and the direct limit theorem for singular homology show that $z \times 0$ in the complement of a $(k-1)$-cell, which is a contradiction.

(b) For each $i \geqq 0$, write the standard $i$-sphere $S^{i}$ as the union of its upper cap $U^{i}$ and its lower cap $L^{i}$, so that $U^{i} \cap L^{i}=S^{i-1}$. Now let $S_{0}^{k}$ be the image of an imbedding $h$ of $S^{k}$ into $M$. For $i \leqq k$, let $S_{0}^{t}$ $=h\left(S^{i}\right), U_{0}^{i}=h\left(U^{i}\right)$, and $L_{0}^{i}=h\left(L^{i}\right)$. Then part (a) and the MayerVietoris sequence of the proper triad $\left(M-S_{0}^{i-1} ; M-U_{0}^{i}, M-L_{0}^{i}\right)$ give the isomorphism

$$
H_{q}\left(M-S_{0}^{i}\right) \approx H_{q+1}\left(M-S_{0}^{i-1}\right) \quad(0 \leqq i \leqq k, 0 \leqq q<n-i) .
$$

Applying this successively, we get the chain of isomorphisms

$$
\begin{aligned}
H_{p}\left(M-S_{0}^{k}\right) & \approx H_{p+1}\left(M-S_{0}^{k-1}\right) \approx \cdots \approx H_{p+k}\left(M-S_{0}^{0}\right) \\
& \approx H_{p+k+1}\left(M-S_{0}^{-1}\right)=H_{p+k+1}(M) .
\end{aligned}
$$

This completes the proof of Theorem 4 .

Now let us give the examples promised after the statement of the theorem. The complement of the $k$-cell $D^{k}$ in the $A^{n}$-space $D^{n+2}$ has the same homotopy type as $S^{n-k+1}$, hence is not $(n-k+1)$-acyclic. It is easy to see from the proof of part (b) that in the critical case $p=n-k$ we always get $H_{n-k}\left(M-S_{0}^{k}\right)$ as a homomorphic image of $H_{n+1}(M)$. To get an example where $H_{n-k}\left(M-S_{0}^{k}\right)=0$ but $H_{n+1}(M)$ $\neq 0$, we may take $M$ to be the $A^{n}$-space $R^{n+2}-0$ with $S_{0}^{k}=S^{k}$.

7. Proof of Theorem 3. The case $n=0$ of the theorem is trivial. Suppose now that $n$ is any positive integer and that $M$ is an $A^{n}$-space with $H_{n}(M) \neq 0$. Let us show that $M$ is a Peano space (locally connected, compact, connected, metrizable space). Let $\{x, y\} \subset M$. There must be a point $w$ in $M$ such that $\{x, y\} \subset M-w$, since $H_{n}(M) \neq 0$. Since $H_{0}(M-w)=0$, there is a path from $x$ to $y$. Thus $M$ is pathwise connected. Now choose a nonbounding $n$-cycle $z=\sum_{i=1}^{r} m_{i} T_{i}$ on $M\left(m_{i}\right.$ an integer, $T_{i}$ a singular $n$-simplex $)$. The carrier $|z|=\bigcup_{i=1}^{r}$ im $T_{i}$ must cover all of $M$, since for each point $x$ of $M, H_{n}(M-x)=0$. Since $M$ is pathwise connected, it is easy then to construct a map of a Peano space (consisting of $r n$-simplexes and $(r-1)$ arcs) onto $M$. But every Hausdorff space which is the continuous image of a Peano space is itself a Peano space (see for instance $[4$, p. 126]). 
Now let us show that every $(n-1)$-sphere $S_{0}^{n-1}$ in $M$ separates $M$. By part (b) of Theorem $4, H_{0}\left(M-S_{0}^{n-1}\right) \approx H_{n}(M) \neq 0$. Hence $M-S_{0}^{n-1}$ has more than one path-component. But the path-components of an open subset of a Peano space are the same as its components [4, p. 118]. It can be shown using part (a) of Theorem 4 that $S_{0}^{n-1}$ is the boundary of each of the components of $M-S_{0}^{n-1}$, but this is not needed in the following.

Case $n=1$. By the preceding, $M$ is a compact, connected metrizable space (containing more than one point, since $H_{1}(M) \neq 0$ ) which is separated by every pair of its points. Hence by $[4$, p. 55] $M$ is homeomorphic to $S^{1}$.

Case $n=2$. Zippin's [10] characterization of the 2-sphere implies that $M$ is homeomorphic to $S^{2}$ provided that (i) $M$ is a Peano space, (ii) every simple closed curve in $M$ separates $M$, (iii) no arc in $M$ separates $M$, and (iv) $M$ contains a simple closed curve. Conditions (i) and (ii) have been established. Condition (iii) follows from Theorem 4 (a) with $n=2$ and $k=1$. If condition (iv) did not hold, then by condition (i), $M$ would be (by definition) a dendrite [8, p. 88]. But a dendrite contains (uncountably many) cut points [8, p. 88]. This contradicts the fact that $M$ is an $A^{2}$-space. The proof of Theorem 3 is complete.

\section{REFERENCES}

1. J. W. Alexander, A proof and extension of the Jordan-Brouwer separation theorem, Trans. Amer. Math. Soc. 23 (1922), 333-349.

2. E. Artin and H. Braun, Vorlesungen über algebraische Topologie, ausgearbeitet von Armin Thedy, Mathematisches Seminar der Universität Hamburg, Hamburg, 1964.

3. S. Eilenberg and N. Steenrod, Foundations of algebraic topology, Princeton Univ. Press, Princeton, N. J., 1952. 1961.

4. J. G. Hocking and G. S. Young, Topology, Addison-Wesley, Reading, Mass.,

5. V. L. Klee, Convex bodies and periodic homeomorphisms in Hilbert space, Trans. Amer. Math. Soc. 74 (1953), 10-43.

6. M. C. McCord, Singular homology groups and homotopy groups of finite topological spaces, Duke Math. J. (to appear).

7. H. Seifert and W. Threlfall, Lehrbuch der Topologie, Chelsea, New York, 1934.

8. G. T. Whyburn, Analytic topology, Amer. Math. Soc. Colloq. Publ. Vol. 28, Amer. Math. Soc., Providence, R. I., 1942.

9. R. L. Wilder, Topology of manifolds, Amer. Math. Soc. Colloq. Publ. Vol. 32, Amer. Math. Soc., Providence, R. I., 1949.

10. L. Zippin, On continuous curves and the Jordan curve theorem, Amer. J. Math. 52 (1930), 331-350.

The University of Georgia 